\title{
Nice tiling, nice geometry!?!
}

\author{
EMil MolnÁR
}

To memory of my father Ernö Molnár, an enthusiastic teacher of mathematics, on the 100th anniversary of his birth

Abstract. The squared papers in our booklets, or the squared (maybe black and white) pavements in the streets arise an amusing problem: How to deform the side segments of the square pattern, so that the side lines further remain equal (congruent) to each other? More precisely, we require that each congruent transformation of the new pattern, mapping any deformed side segment onto another one, leaves the whole (infinitely extended) pattern invariant (unchanged).

It turns out that there are exactly 14 types of such edge-transitive (or so-called isotoxal) quadrangle tilings, sometimes with two different forms (e.g. black and white) of quadrangles (see Figure 2). Such a collection of tiling can be very nice, perhaps also useful for decorative pavements in streets, in flats, etc.

I shall sketch the solution of the problem that leads to fine (and important) mathematical concepts (as barycentric triangulation of a polygonal tiling, adjacency operations, adjacency matrix, symmetry group of a tiling, $D$-symbol, etc). All these can be discussed in an enjoyable way, e.g. in a special mathematical circle of a secondary school, or in more elementary form as visually attractive figures in a primary school as well.

My colleague, István Prok [11] developed an attractive computer program on the Euclidean plane crystallographic groups with a nice interactive play (for free download), see our Figures $3-5$.

A complete classification of such Euclidean plane tilings (not only with quadrangles) can be interesting for university students as well, hopefully also for the Reader (Audience). This is why I shall give some references, where you find also other ones.

Further problems indicate the efficiency of this theory now. All these demonstrate the usual procedure of mathematics and the (teaching) methodology as well: We start with a concrete problem, then extend it further, step-by-step by creating new manipulations, concepts and methods. So we get a theory at certain abstraction level. Then newer problems arise, etc.

This paper is an extended version of the presentation and the conference paper [7].

The author thanks the Organizers, especially their head Professor Margita Pavleković for the invitation, support and for the kind atmosphere of the conference.

Copyright (C) 2012 by University of Debrecen 


$$
\text { "tmcs-molnar" — 2012/11/24 — 17:56 — page } 270 — \text { — } 2
$$

Key words and phrases: tiling, barycentric subdivision, symmetry group, D-symbol, computer graphics, algorithmic classification, teaching mathematics through activities.

ZDM Subject Classification: G99.

\section{Introduction}

After a longer experimental period, when dealing with a collection of squared papers, the school pupils (students) will get good and erroneous constructions in individual and collective work as well. They can conclude (probably in the second or third occasion, step-by-step, under direction of the teacher, as little as possible) to the following really fine idea (being described now in a compact form [4]):

Our Figure 1 shows the usual square tiling with its 2-dimensional face centers indicated by small triangles $\Delta$, with (1-dimensional) edge centers denoted by $\diamond$, and (0-dimensional) vertices $\circ$. The opposite full - , dashed --- and dotted ..... lines, respectively, are introduced as well, so that we obtain the so-called barycentric subdivision of the square tiling into barycentric triangle tiling denoted by $C$. That is important in the description of our simple square tiling (moreover of any polygonal tiling, furthermore of any 'topological' one; with corresponding changes, of course [2]).

4 barycentric triangles $(1,2,3,4$ in Figure 1 top left) support to any edge — from both sides. They form the so-called edge domain VPWQ, playing an important role in description of the possible edge modifications. Namely, an edge can be drawn almost freely between the vertices $V$ and $W$ in the edge domain $V P W Q$. Then a plane group (as a rule of periodicity) with the starting edge domain as fundamental domain (repetitive unit) will tile the whole square tiling, with the modified edge in it (see also Figure 2: 2.3, 2.4, 2.5, 3.2, 3.3, 3.4).

But the modified edge can also be symmetric with respect to its midpoint (right bottom of Figure 1, and in Figure 2: 1.3, 1.5). Or it is symmetric w.r.t. the side edge itself (left bottom of Figure 1, and Figure 2: 1.2, 1.4). Here the edge cannot change, but extra label (mark), in the half edge domain, shows the situation. Or it is symmetric w.r.t. the midline of the side (Figure 1 top right, and Figure 2: 2.1, 3.1). Or it is symmetric in both of the last mirror lines (Figure 1 top middle, and Figure 2: 1.1), this is just the original square tiling.

The remaining task to solve is how to display these edge domains in a regular way (as formulated in the Abstract), so that they form a 'topological 
quadrangle tiling' in the plane, and to any two new edges there exists an isometry of the plane that maps the first edge onto the second one and the whole plane tiling will be mapped onto itself.

For this last step we would need the classification of the 17 plane crystallographic groups, as the program [11] shows, or at least to overview those possibilities, how the edge domain can be a fundamental domain (repetitive unit) for a tiling with the edge domains above? This task needs only finitely many side pairings of this quadrangle edge domain and its parts above (Figure 1-2).

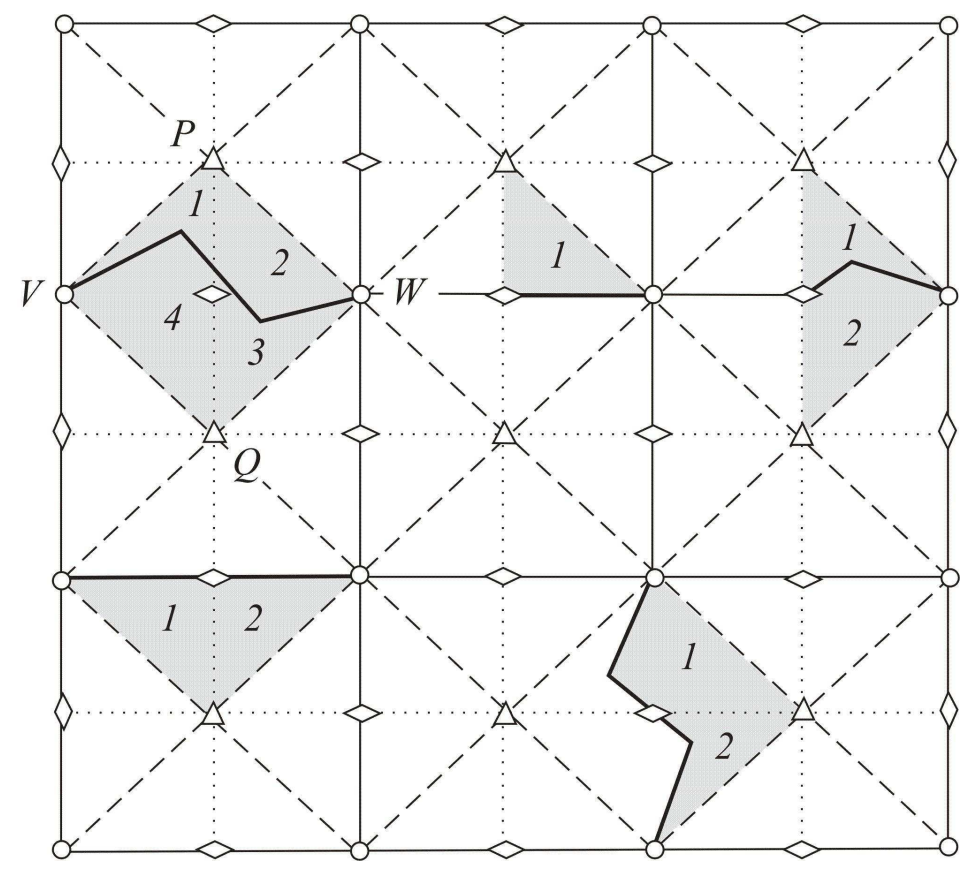

Figure 1. The square tiling and its barycentric subdivision. Edge domain $V Q W P$ of 4 barycentric triangles. Symmetries of the edge domain yield smaller fundamental domains.

That means, this problem provides also a theoretic benefit by offering insight into some plane crystallographic groups ( 7 out from 17). This could be an attractive experiment for an ambitious teacher in the special mathematical circle of a secondary school, or also of a primary one.

I collected in Figure 2 the complete list of the solutions: the 14 types of our "edge-transitive" (isotoxal) quadrangle tilings as illustration from [4], where the 
more general scientific problem on spatial cube tilings has also been solved. The Reader finds also a plane generalization in [2].

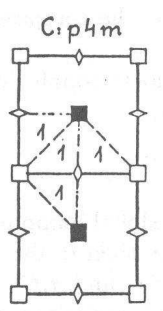

1.1

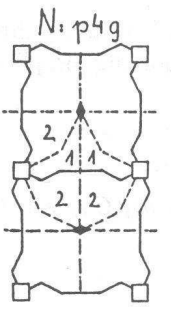

2.1

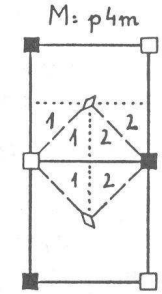

1.2

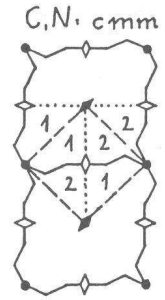

1.3

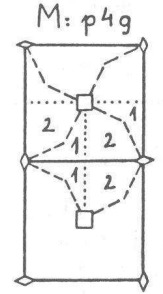

1.4

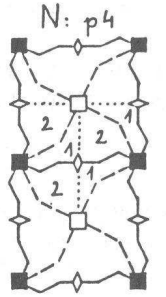

1.5

$N: c m$

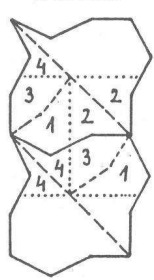

2.3

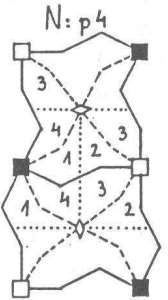

2.4

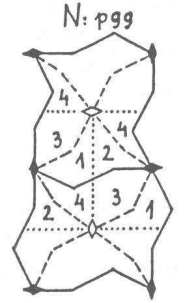

2.5

$N: p 4 m$
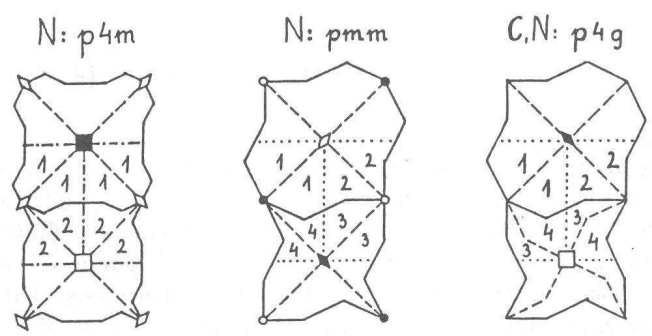

N: $p 4$
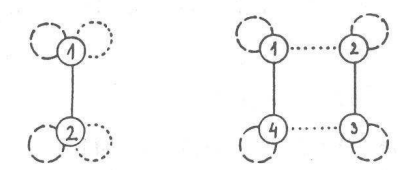

3.2<smiles></smiles>

3.3

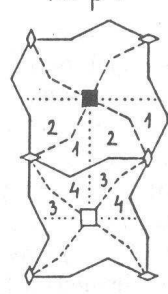<smiles>C1COCCO1</smiles>

3.4

Figure 2. The 14 families of quadrangle tilings with equal edges:

1. Equal tiles with edge symmetry; 2 . Equal tiles with vertex symmetry; 3 . Non-equal (black and white) tiles with $D$-diagrams. 


\section{The solution}

Our Figure 2 shows the 14 types of edge-transitive square tilings by at most two typical tiles with the edge domains of numbered barycentric triangles. We have divided the list into 3 classes.

Class 1 contains five members 1.1-1.5 where the symmetry group of the edge (i.e. the stabilizer group of the edge center) maps the two adjacent tiles to each other.

Class 2 also consists of five tilings 2.1-2.5 where we have also equal tiles not by the edge stabilizer but by the vertex one.

Class 3 has four surprising tilings 3.1-3.4 with two types (e.g. with black and white colors) of tiles not mapped to each other, despite all the edges are mapped onto each other.

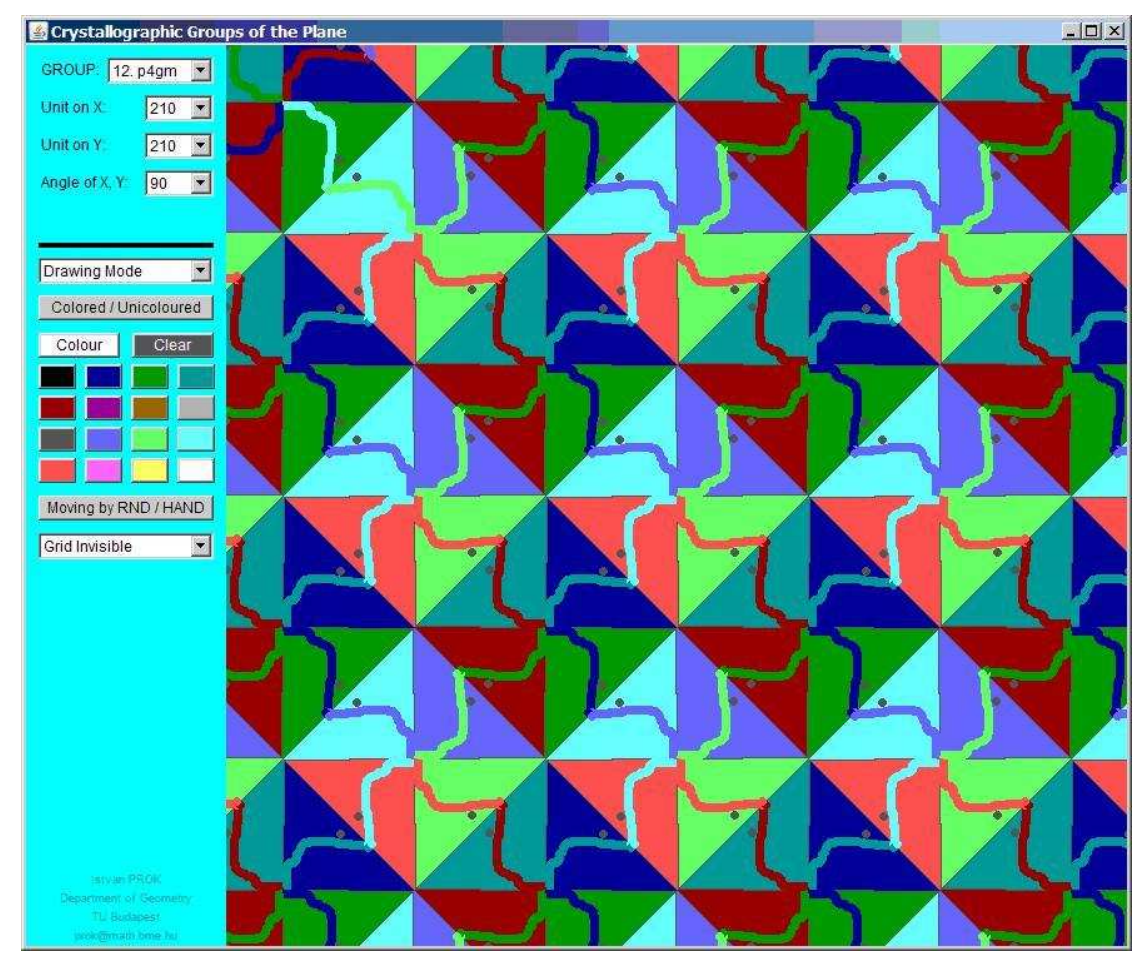

Figure 3. I. Prok's program [11] provides a drawing mode with various colors. It makes black and white pictures as well.

http://www . math. bme.hu/ prok 


$$
\text { "tmcs-molnar" — 2012/11/24 - 17:56 — page } 274 \text { — \#6 }
$$

At each tiling in Figure 3 we have indicated by an initial capital letter, whether a convex $(C)$, or a non-convex $(N)$ realization (sometimes both ones, think of!) is (are) possible. $M$ indicates that marks are necessary for "symmetry breaking". Moreover, you find the crystallographic plane group to each tiling in international denotation (7 groups out of 17$)$.

\section{On D-symbols. Illustration with computer pictures}

You find also the very important adjacency diagram (adjacency graph) to the so-called D-symbol $\mathcal{D}\left(D, \Sigma_{I}, M\right)$. (for initiative of B. N. Delone (Delaunay), M. S. Delaney, A. W. M. Dress) at class 3. Find the other ones to the classes 1-2, please! Note that each diagram (graph) $\mathcal{D}$ remains connected if we exclude the dashed - - - lines from the diagram. This assertion will be the criterion of edge-transitivity of a plane tiling!

It is a very nice idea, isn't it!?

This would be the next level of abstraction, mainly for university students or to audience of a scientific conference. Of course, this is only to indicate an important method for research at a scientific level for other tilings in the plane; or - much more important and with new scientific results (e.g. in [1], [2], [4], [5], [6]) - in a homogeneous space (e.g. one of constant curvature), where many problems are still open.

For each D-diagram $\mathcal{D}$ in Figure 2: $3.1-3.4$ we consider the barycentric triangle subdivision $C$ (Figure 1) of the square tiling. Any such triangle of the edge domain $(1,2,3,4$ or 1,2 or 1 , respectively) will be indicated by a vertex of the diagram $\mathcal{D}$. The dotted $\cdots \cdots$ lines, now seen as $\sigma_{0}$ operation of $C$ and of $\mathcal{D}$, the dashed - - lines as $\sigma_{1}$ operation of $C$ and of $\mathcal{D}$, the full — lines as $\sigma_{2}$ operation of $C$ and of $\mathcal{D}$ indicate the adjacencies of the diagram $\mathcal{D}$ (with the above labeled or colored edges of graph $\mathcal{D}$ ) according to the common sides of the barycentric triangles in $C$.

Please observe that the images of the edge domains or smaller fundamental domains in Figure 1-2 will also be induced by these adjacency operations.

That means that the whole symmetry group $\Gamma$ of the tiling will be described if we give the (symmetric) so called adjacency matrix function $M=$ $\left(m_{i j}\right)$ of diagram $\mathcal{D}$. The entry $m_{i j}$ shows, how many barycentric triangles of side $i$ and $j$ (the half of them) surround a $k$-vertex of $C,\{i, j, k\}=\{0,1,2\}$, according to the corresponding ij-path in $\mathcal{D}$. That means that each element (vertex) of 


$$
\text { "tmcs-molnar" — 2012/11/24 - 17:56 — page } 275 \text { — \#7 }
$$

diagram $\mathcal{D}$ describes the corresponding triangle class (orbit) of $C$ by the symmetry group $\Gamma$, so that $\Gamma$ preserves the adjacencies of $C$.

Now the adjacency matrix function $M$ is constant: $\left(\begin{array}{lll}1 & 4 & 2 \\ 4 & 1 & 4 \\ 2 & 4 & 1\end{array}\right)$, i.e. the same for each element of $\mathcal{D}$ and of $C$, because of the square tiling or quadrangle tiling, where 4 quadrangles meet at each vertex.

Consider e.g. Figure 2: 3.3 with its picture and its diagram, where the adjacency operations can be given by involutive (involutory) permutations (in general) as follows

$\sigma_{0}:(12)(34) ; \sigma_{1}:(1)(2)(34)$ as the loops show it at vertex 1 and $2 ; \sigma_{2}:(14)(23)$.

Now $\left(\sigma_{1}, \sigma_{0}\right)$ at the tile centers (2-centers) of triangles 1,2 in $C$ shows the dihedral corner for $\Gamma ;\left(\sigma_{1}, \sigma_{0}\right)$ at tile centers of triangles 3, 4 shows the 4-rotation in $\Gamma$ by matrix entries $m_{01}=m_{10}=4$ (please check them carefully!). The $\left(\sigma_{2}, \sigma_{1}\right)$ composition (always in opposite order by our convention now) provides the line reflection at the common triangle vertices (0-centers) of the triangles $1,2,3,4$ in $C$, again by matrix entries $m_{12}=m_{21}=4$ (check it, please!). The $\left(\sigma_{2}, \sigma_{0}\right)$ composition provides the trivial stabilizer (the identity) in $\Gamma$ at the edge centers (1-centers, 1 -vertices) of triangles $1,2,3,4$ by matrix entries $m_{02}=m_{20}=2$. Thus the edge domain provides a fundamental domain for the plane group $\mathbf{p} 4 \mathbf{g}$, indeed. This procedure can have an axiomatic or algorithmic formulation and it can be computerized as well (e.g. as in our paper [1]). See also the Appendix at the end of this paper.

Another edge-transitive tiling to $\Gamma=\mathbf{p} 4 \mathrm{~g}=\mathbf{p} 4 \mathbf{g m}$ is pictured by computer program [11] in Figure 3 (also by Figure 2:2.1), where we have drawn an edge motif into a fundamental domain of the colored picture which can be very attractive and amusing.

The method is general, we can draw all the 14 types by this strategy, and also other Euclidean or non-Euclidean isotoxal tilings (see [2]).

Finally, you see two black and white computer illustrations by the author (Figure 4-5). The first picture is drawn with the former pattern by the group $\Gamma=\mathbf{p} 4 \mathbf{g}$ (Figure 4 ). The second one is by the group no. $8 \mathbf{p} 2 \mathbf{g g}=\mathbf{p g g}$ (please, find it also in Figure 2).

\section{Please, continue drawing!!!}

I thank my colleague Dr. Jenö Szirmai for his help in preparing the manuscript, I also thank the unknown referees for their valuable suggestions. 


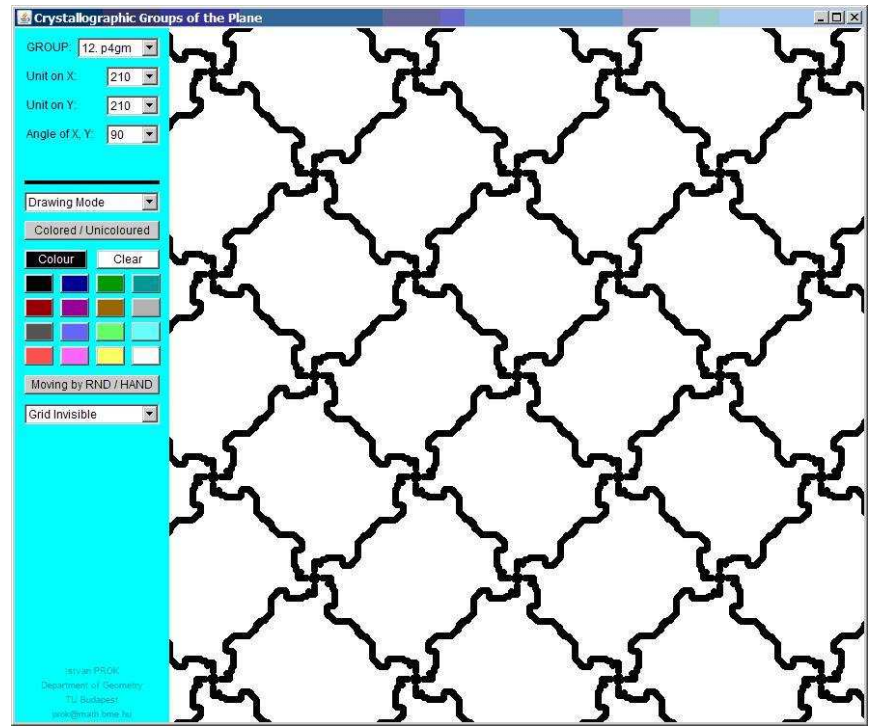

Figure 4. Black and white pattern by the former plane group $\mathbf{p 4 g m}$ no. 12 .

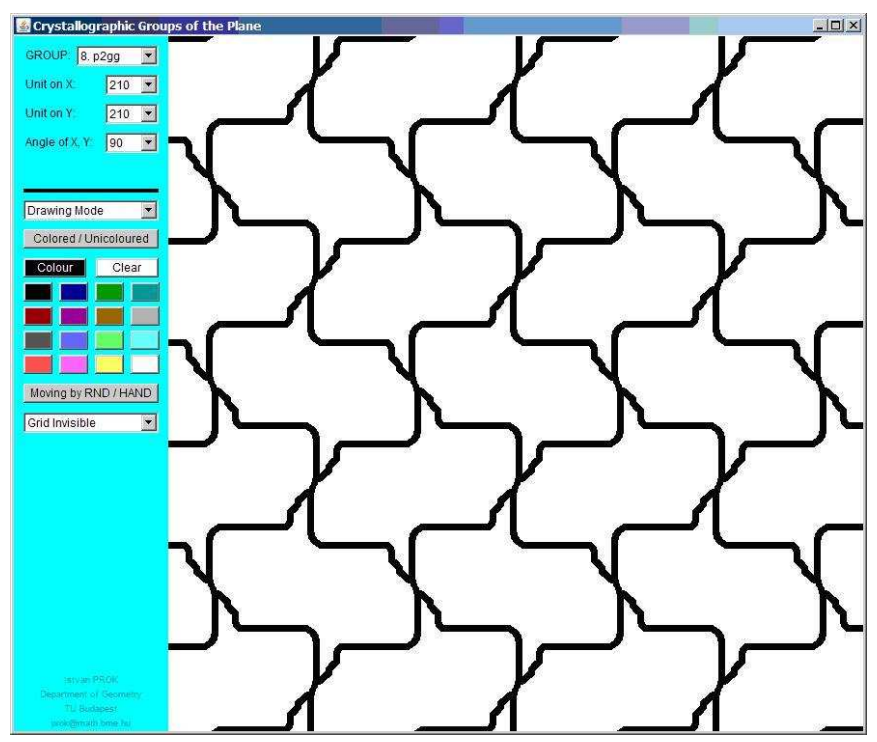

Figure 5. Pattern by plane group p2gg no. 8 . 


$$
\text { "tmcs-molnar" — 2012/11/24 — 17:56 — page } 277 \text { — \#9 }
$$

\section{Appendix for interested readers. Axioms for $D$-symbols, some problems}

After the previous visual description, we formulate a system of axioms for the D-symbol structure $\mathcal{D}\left(D, \Sigma_{I}, M\right)$. (For details see [1], [4], [5], [6], [8], moreover [9], [10], [12] for other aspects and applications).

Here $D=\left\{D_{1}, D_{2}, \ldots D_{n}\right\}$ is a set of finite, say of $n$ elements ( $n \in \mathbb{N}=$ $\{1,2, \ldots\}$ the natural numbers) with $|I|=d+1$ involutive (involutory) adjacency operations $\Sigma_{I}=\left\{\sigma_{0}, \sigma_{1}, \ldots, \sigma_{d}\right\}$ on $\mathcal{D}$ (each acts as permutation, say from the left on $\mathcal{D})$.

- Think of the plane, i.e. $d=2$, and of a fundamental polygon (domain) $F$ as before - glued together of $n$ barycentric triangles (or simplices in dimensions d). This logical gluing is just by the adjacency operations $\sigma_{i}$, each along the $i$-sides (side-facets), $i \in I=\{0,1, \ldots, d\}$, while the gluing is possible, according to the axioms below.

The free (not glued yet) $j$-sides (side-facets) generate a group $\Gamma$. Now each generator will be a mapping ${ }^{j} g$ (occasionally with further numbering subindex on the left of $g$ ) which maps this free $j$-side onto the corresponding free $j$-side, and ${ }^{j} g$ maps the triangle (simplex) of $F$ along the above $j$-side onto the image triangle along the image $j$-side of the image fundamental domain $F .{ }^{j} g, j$-adjacent with $F$. An analogous argumentation defines the inverse generator ${ }^{j} g^{-1}$, so the side pairing of $F$. Involutive (involutory) generator can also occur, e.g. reflection in a side (-facet) of $F$, etc.

In this sense, while the adjacency operations of $\Sigma_{I}$ acts from the left on $\mathcal{D}$ and on $F$, the transformation group $\Gamma$ acts from the right on $F$ and on its $\Gamma$ images. The parentheses in the formula

$$
\left(\sigma_{i} D\right) \cdot{ }^{j} g=\sigma_{i}\left(D \cdot{ }^{j} g\right)
$$

express this formal associativity law, and we can say: The fundamental group $\Gamma$ of the tiling, with the $\Gamma$ images of domain $F$, preserves the adjacency relations of the barycentric simplices (previously denoted by $C$ ), of the fundamental tiling denoted by $F \Gamma$.

Now the $\mathbb{N}$ valued symmetric matrix function $M: D \rightarrow \mathbb{N}_{I \times I}, D \rightarrow m_{i j}(D)$ will determine the branching numbers at $(0=(d-2)$-dimensional) vertices (edges for $d=3$ ) at the intersection of $i j$-sides of the barycentric triangles (simplices), so at the fundamental domain $F$ in $F \Gamma$. These will serve the so-called defining relations for the above generators ${ }^{j} g$ of $\Gamma$ (see the crucial Axiom iii) below). 


$$
\text { "tmcs-molnar" — 2012/11/24 — 17:56 — page 278 — \#10 }
$$

The axioms will be expressed by the above matrix function $M$ as follows:

i) $m_{j j}(D)=1$

ii) $m_{i j}\left(\sigma_{i} D\right)=m_{i j}(D)=m_{j i}(D)$

iii) $\left(\sigma_{j} \sigma_{i}\right) \ldots m_{i j}(D)$ times $\ldots\left(\sigma_{j} \sigma_{i}\right) D=D$

iv) $m_{j k}(D)=2$ if $|j-k|>1$

v) if $|j-k|=1$, then $m_{j k}$ ( $D>2$ (or $\geq 2$ for generalization, e.g. for digons on the ordinary sphere $\mathbb{S}^{2}$ ).

All these hold for any $D \in \mathcal{D}$ and for indices $i, j, k \in I$.

Such a concise formulation can give us a good esthetical feeling, that mathematics and geometry are nice! Although this level of abstraction, now far from being visual, might be strange enough? But think of that our starting problem and its generalization on the sphere, on the Bolyai-Lobachevskian hyperbolic plane, each of them satisfies these axioms. We can say: the structure $\mathcal{D}\left(D, \Sigma_{I}, M\right)$ has a lot of models! By these signs !?! we have also referred to the title of this paper, again.

We recall e.g. from [1] or [8] the so-called curvature formula for $d=2$ dimensional $D$-symbols

$$
\begin{array}{rlrl}
K(\mathcal{D}):=\sum_{D \in \mathcal{D}}\left(1 / m_{01}(D)+1 / m_{02}(D)+1 / m_{12}(D)-1\right) & =0 & \mathbb{S}^{2} \\
& < & \mathbb{E}^{2} \\
\mathbb{H}^{2}
\end{array}
$$

as a general theorem of the structure $\mathcal{D}\left(D, \Sigma_{I}, M\right)$ completely characterizes the corresponding tiling: Whether it is realizable on the sphere $\mathbb{S}^{2}(>0)$ for so-called "good orbifolds" which means roughly that the opposite points of the sphere have the same stabilizer group, respectively (see e.g. [2]). Whether it is realizable in the Euclidean plane $\mathbb{E}^{2}(=0)$, or in the Bolyai-Lobachevskian hyperbolic plane $\mathbb{H}^{2}(<0)$. Moreover, this formula can also be applied for higher dimensional $D$-symbols to other index triples.

E.g. for $d=3$, we consider the most important application, the spatial tilings in the crystallography. Then the so-called partial $D$-symbol $\mathcal{D}^{3}\left(D, \Sigma_{I \backslash 3}, M^{3}\right)$, i.e. we exclude the $\sigma_{3}$ operation and the corresponding matrix entries, the remaining 2-dimensional $D$-symbol components will characterize the 3-centre figures of the solid(s) of the hypothetic tiling, might belong to the original $D$-symbol $\mathcal{D}\left(D, \Sigma_{I}, M\right)$. In the case of a proper 3-tiling these components each has to describe an $\mathbb{S}^{2}$ tiling, easy to check by the above curvature formula. But similar 


$$
\text { "tmcs-molnar" — 2012/11/24 — 17:56 — page } 279 \text { — \#11 }
$$

criteria hold for the vertex figures, where the $\sigma_{0}$ operation will be excluded from the original 3-dimensional $D$-symbol. Any proper vertex will be described by an $\mathbb{S}^{2}$ tiling, again.

We have just arrived to the main problem of D-symbols: whether a well-formed symbol (satisfying the axioms) can be realized by a tiling in an appropriate space or not. In dimensions $d=2$ the above curvature formula gives complete information (in case of $\mathbb{S}^{2}$ the good orbifolds can easily be characterized). But for dimensions $d=3$ only partial results are known.

E.g. in the joint work [1] we have described all the 88 face-transitive tilings of the Euclidean space $\mathbb{E}^{3}$ by (marked) topological polyhedra. Our computer program provided the possibilities, where the 3 -dimensional $D$-symbol had the partial $D$-symbol $\mathcal{D}^{2}\left(D, \Sigma_{I \backslash 2}, M^{2}\right)$ having only one component (this guaranteed just the face-transitivity). Then the realizations were checked (not easily) by the 219 (known) isomorphism classes of the crystallographic groups of $\mathbb{E}^{3}$.

With my doctor students Rita Kós and Lajos Boróczki we are working on the extensions of this problem circle to non-Euclidean geometries.

The situations are well-described in our summarizing paper [6], where besides the classical spaces $\mathbb{S}^{3}, \mathbb{E}^{3}, \mathbb{H}^{3}$ of constant curvature other 5 Thurston geometries $\mathbb{S}^{2} \times \mathbb{R}, \mathbb{H}^{2} \times \mathbb{R}, \sim \mathbb{S L}_{2} \mathbb{R}, \mathbf{N i l}$ and Sol also come into the game [8].

But now we are far from the starting problem of the paper !?!

\section{References}

[1] A. W. M. Dress, D. H. Huson and E. Molnár, The classification of the face-transitive periodic three-dimensional tilings, Acta Crystallographica A49 (1993), 806-817.

[2] Z. Lučić, E. Molnár and M. Stojanović, The 14 infinite series of isotoxal tilings in the planes of constant curvature, Periodica Math. Hung. 29(2) (1994), 177-195.

[3] E. Molnár, Díszítések és minták (M. C. Escher stílusában D-szimbólumokkal), "Új utak és lehetőségek a geometriában." Nagykanizsa, 1993 október 13-16, Matematikai Lapok, Új sorozat 3. évf. (1993)(1-2), 17-37, (1996).

[4] E. Molnár, Symmetry breaking of the cube tiling and the spatial chess board by D-symbols, Beiträge zur Algebra und Geometrie (Contr. Alg. Geom.) 35, no. 2 (1994), 205-238.

[5] E. Molnár, Discontinuous groups in homogeneous Riemannian spaces by classification of D-symbols, Publ. Math. Debrecen 49/3-4 (1996), 265-294.

[6] E. Molnár, I. Prok and J. Szirmai, Classification of solid transitive simplex tilings and their realizations in homogeneous spaces, Non-Euclidean Geometries, János Bolyai Memorial Volume, Vol. 581, Mathematics and Its Applications, (A. Prékopa and E. Molnár, eds.), Springer, 2006, 321-363. 


$$
\text { "tmcs-molnar" — 2012/11/24 — 17:56 — page } 280 \text { — \#12 }
$$

[7] E. Molnár, Nice tilings, nice mathematics!?!, The 3rd International Scientific Colloquium MATHEMATICS AND CHILDREN (The Math. Teacher), Osiek/Croatia, March 18-19 2011, pp. 113-119 and 381-387 (in Hungarian).

[8] E. Molnár, On D-symbols and orbifolds in an algorithmic way, Atti Semin. Mat. Fis. Univ. Modena Reggio Emilia 58 (2011), 263-276.

[9] E. Molnár and J. Szirmai, Symmetries in the 8 homogeneous 3-geometries, Symmetry: Culture and Science 21, no. 1-3 (2010), 87-117.

[10] E. Molnár, J. Szirmai and J. R. Weeks, 3-simplex tilings, splitting orbifolds and manifolds, Symmetry: Culture and Science 22, no. 3-4 (2011), 435-458.

[11] I. Prok, Crystallographic Groups of the Plane (for free download), http://www. math. bme.hu/ prok.

[12] J. Szirmai, Über eine unendliche Serie der Polyederpflasterungen von flächentransitiven Bewegungsgruppen, Acta Math. Hungar. 73, no. 3 (1996), 247-261.

EMIL MOLNÁR

INSTITUTE DF MATHEMATICS, DEPARTMENT OF GEOMETRY

BUDAPEST UNIVERSITY OF TECHNOLOGY AND ECONOMICS

H-1521, BUDAPEST

EGRY JOZSEF U. 1. H II. 22.

E-mail: emolnar@math.bme.hu

(Received August, 2011) 